\title{
Pancreatic Tail Pseudocyst Mimicking Adrenal Lesion during Laparoscopic Left Adrenalectomy for Pheochromocytoma
}

\author{
Man H Tang ${ }^{1}$, Anil D Rao², Reyaz M Singaporewalla ${ }^{3}$
}

\begin{abstract}
Aim and objective: This report highlights a case of a pancreatic tail pseudocyst mimicking an adrenal lesion during laparoscopic adrenalectomy for left-sided pheochromocytoma.

Background: Minimally invasive adrenalectomy has become the gold standard for surgical excision for most adrenal lesions. There are, however, potential complications including injury to surrounding vital structures related to such minimally invasive procedures and can result in devastating consequences. Excellent knowledge of local anatomy with imaging correlation is mandatory to avoid complications.

Case description: A 59-year-old woman presented initially with severe pancreatitis and was found to have a left adrenal incidentaloma on crosssectioning imaging. Subsequent functional workup was suggestive of a pheochromocytoma and she underwent laparoscopic left adrenalectomy. Intraoperatively, a lesion in close proximity to the left kidney was initially thought to be the adrenal lesion but was found to be a pseudocyst arising from the tail of the pancreas instead upon further dissection. The left adrenal tumor was eventually resected without any complications. Conclusion: The pancreatic tail lesion can be mistaken to be a left adrenal tumor during laparoscopic left adrenal surgery.

Significant findings: This case again highlights the importance of careful dissection of surgical planes and preoperative assessment of imaging for endoscopic adrenalectomy.

Keywords: Adrenalectomy, Pancreatic pseudocyst, Pheochromocytoma.

World Journal of Endocrine Surgery (2021): 10.5005/jp-journals-10002-1319
\end{abstract}

Knowledge of the retroperitoneal anatomy is important while performing laparoscopic adrenalectomy. Pancreatic injury is associated with transperitoneal left adrenalectomy due to their close proximity. We present an image of interest of a surgical case that depicts how a pancreatic tail pseudocyst can be mistaken as the left adrenal gland during laparoscopic adrenalectomy. The patient initially presented with severe pancreatitis and a left adrenal lesion detected on imaging, which was confirmed to be a pheochromocytoma.

Intraoperatively, there was a lack of any blood pressure fluctuations that we would usually expect when mobilizing a pheochromocytoma. Visualization of the retroperitoneal area was also suboptimal due to the significant amount of visceral fat tissue and the patient's body habitus. A repeat intraoperative review of CT images showed the presence of a small pseudocyst that was not noted at the first CT review. We realized that the patient's tail of the pancreas had descended more inferiorly with a formation of a pseudocyst that was lying near the adrenal mass. The adrenal mass was only identified as we traced it superiorly from the superior pole of the kidney.

This case highlights the potential pitfalls faced especially while operating in the left retroperitoneal area. Dissection in the wrong plane or structure can lead to significant
${ }^{1-3}$ Department of General Surgery, Khoo Teck Puat Hospital, Singapore Corresponding Author: Man H Tang, Department of General Surgery, Khoo Teck Puat Hospital, Singapore, Phone: +6596735362, e-mail: manhon_86@hotmail.com

How to cite this article: Tang MH, Rao AD, Singaporewalla RM. Pancreatic Tail Pseudocyst Mimicking Adrenal Lesion during Laparoscopic Left Adrenalectomy for Pheochromocytoma. World J Endoc Surg 2021;13(1):35-36.

Source of support: Nil

Conflict of interest: None

morbidities. We would also like to emphasize the importance and shortcomings of accurate preoperative CT review while planning laparoscopic adrenalectomy. There is a known significant overlap in CT findings for various retroperitoneal lesions, making it difficult to consolidate a diagnosis before biopsy or excision. In addition, identifying critical structures intimate with a target lesion, such as the tail of the pancreas or splenic artery is imperative for surgical planning and ensuring patient safety (Figs 1 and 2).

(c) The Author(s). 2021 Open Access This article is distributed under the terms of the Creative Commons Attribution 4.0 International License (https://creativecommons. org/licenses/by-nc/4.0/), which permits unrestricted use, distribution, and non-commercial reproduction in any medium, provided you give appropriate credit to the original author(s) and the source, provide a link to the Creative Commons license, and indicate if changes were made. The Creative Commons Public Domain Dedication waiver (http://creativecommons.org/publicdomain/zero/1.0/) applies to the data made available in this article, unless otherwise stated. 

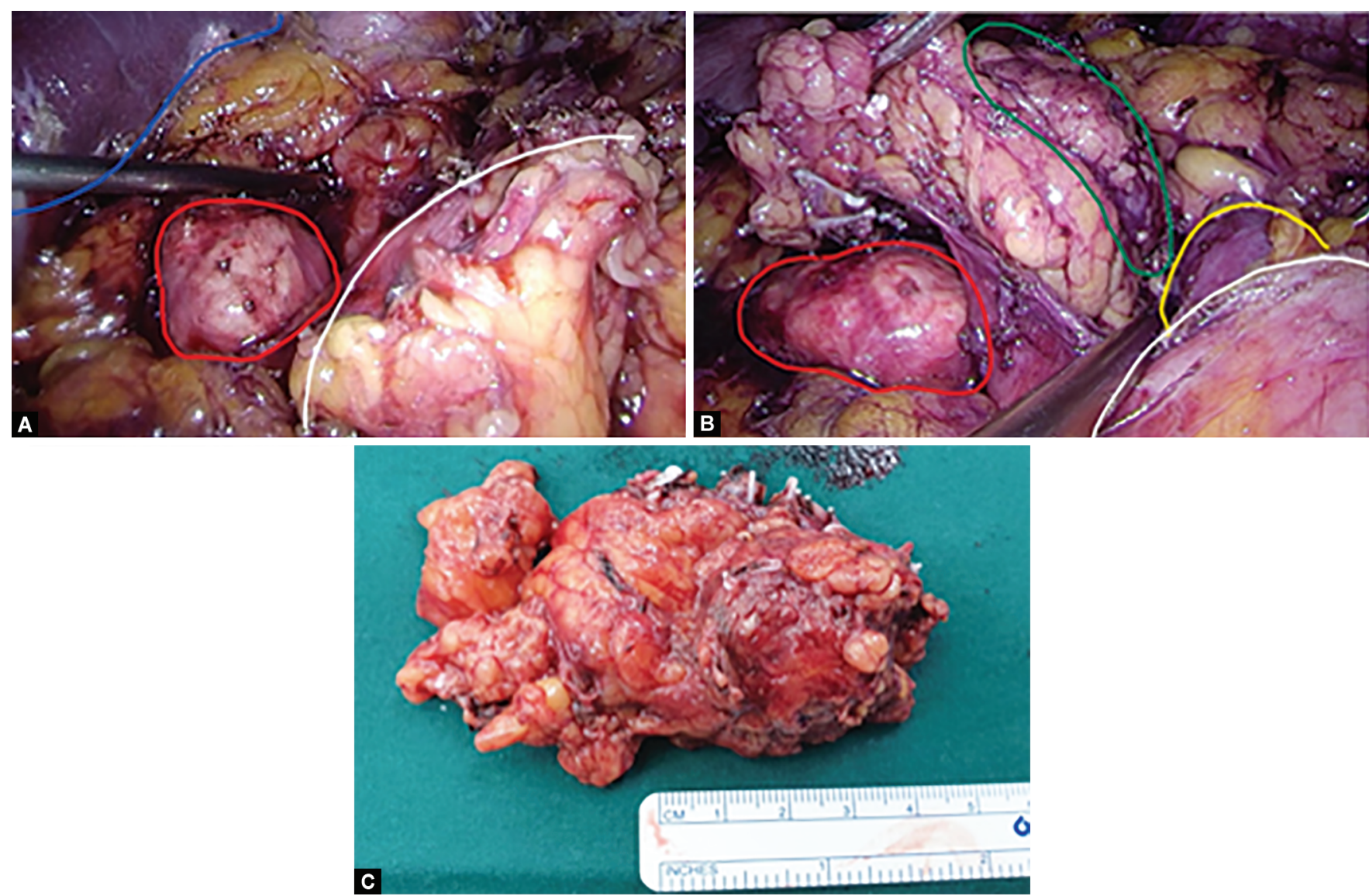

Figs 1 A to C: (A) Intraoperative view of pancreatic pseudocyst (red) initially thought to be adrenal tumor seen in relation to the spleen (blue) after dissection of splenorenal and colorenal ligaments and retraction of Gerota's fascia (white) and retroperitoneal fat tissue; (B) Intraoperative view revealing the adrenal tumor (green) lying superior to the superior pole of the kidney (yellow) in close relation to the pancreatic pseudocyst (red) after further dissection of the Gerota's fascia (white); (C) Final resected specimen of the left adrenal lesion

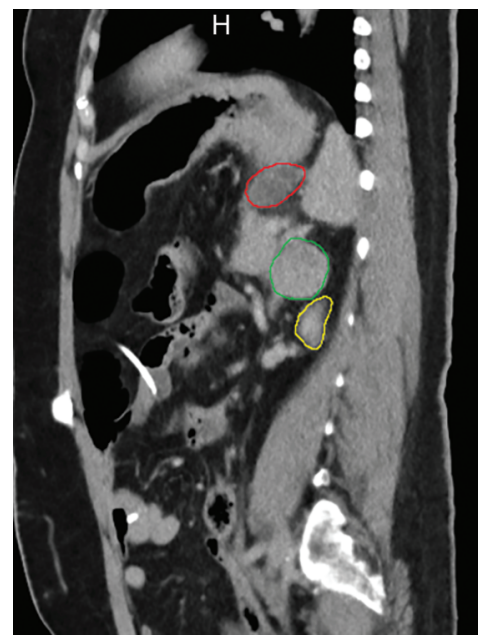

Fig. 2: Sagittal view of CT abdomen and pelvis demonstrating the relation of the left adrenal tumor (green) with the left kidney (yellow) and the pancreatic pseudocyst (red) 